\title{
On Unstability of Certain Group of ODEs with Quasiderivatives
}

\author{
O. PALUMBÍNY
}

\begin{abstract}
The article is focused on the unstability of some group of 4-th ODEs with quasi-derivatives. A sufficient condition of the unstability is followed by proper example.

Mathematics Subject Classification 2000: 34D20

Additional Key Words and Phrases: Fourth-order differential equation, Liapunov, unstability, quasiderivative
\end{abstract}

\section{INTRODUCTION}

The article is a continuation of [2], where some kind of the stability criterion has been derived for the equations (E) with quasiderivatives (the equations (E) as well as the quasiderivatives see below).

In [2] it was also shown an impossibility of replacement (in general) of the quasi-derivatives by ordinary derivatives in differential equations. Therefore new criteria of stability (as well as unstability) are needed.

The aim of the paper is to investigate the unstability (in Liapunov sense) of arbitrary solutions of equations of the form

$$
Q_{4} y+R_{3}(t) Q_{3} y+R_{2}(t) Q_{2} y+R_{1}(t) Q_{1} y+R_{0}(t) Q_{0} y=F(t, y),
$$

where (a prime means a derivative by $t$ )

$$
\begin{gathered}
Q_{0} y(t)=y(t), \quad Q_{i} y(t)=r_{i}(t)\left(Q_{i-1} y(t)\right)^{\prime}, i=1,2,3, \quad Q_{4} y(t)=\left(Q_{3} y(t)\right)^{\prime}, \\
r_{k}(t), k=1,2,3, \quad R_{j}(t), \quad j=0,1,2,3
\end{gathered}
$$

are continuous functions on $I_{b}=[b, \infty), b \in R, F(t, y)$ is continuous on $I_{b} \times R$, where $\quad R=(-\infty, \infty)$. The expressions $Q_{n} y(t), n=0,1,2,3,4$ are called quasiderivatives. 
Let us consider ODE-system (“ODE” means “Ordinary Differential Equation”)

$$
\mathscr{H}_{i}=f_{i}\left(t, y_{1}, y_{2}, y_{3}, y_{4}\right), \quad i=1,2,3,4 \text {. }
$$

MAIN ASSUMPTION. Let (S) be rewritten by vectors as $\&=\mathbf{f}(t, \mathbf{y})$. Let there exists $c$ (real or $-\infty)$ and an area $A \subset R^{4}, \mathbf{o} \in A, \mathbf{o}=(0,0,0,0)$, such that $\mathbf{f}$ is continuous on an area $B=(c, \infty) \times A$ and for any couple $(s, \mathbf{m}) \in B$ the initial-value problem

$$
\mathbf{y}=\mathbf{f}(t, \mathbf{y}), \quad \mathbf{y}(s)=\mathbf{m},
$$

has the only one solution. Let $\mathbf{f}(t, \mathbf{o})=\mathbf{o}$ for all $t>c$.

We shall define (by usual way) the concept of Liapunov stability of the trivial solution of (S) (for more details see Definition 1 in [2]) as well as Liapunov instability of the trivial solution of (S) (Definition 2 in [2]).

Let us consider the differential equation

$$
\begin{aligned}
Q_{4} w(x)+R_{3}(x) Q_{3} w(x)+R_{2}(x) Q_{2} w(x)+R_{1}(x) Q_{1} w(x)+ \\
+
\end{aligned}
$$

as well as the differential system

$$
\begin{aligned}
& w_{i}^{\&}=w_{i+1} / r_{i}(x), i=1,2,3, \\
& \text { \& }_{4}=F\left(x, u(x)+w_{1}\right)-F(x, u(x))-R_{0}(x) w_{1}-R_{1}(x) w_{2}-R_{2}(x) w_{3}-R_{3}(x) w_{4}
\end{aligned}
$$

One can easily see that $w_{1}(t)$ solves $(\mathrm{N})$ iff $\left(z_{1}(t), L_{1} z_{1}(t), L_{2} z_{1}(t), L_{3} z_{1}(t)\right)$ solves $(\mathrm{T})$

("iff" means the usual abbreviation of "if and only if").

We can also state the stability of the trivial solution of $(\mathrm{N})$ by means of the stability of the trivial solution of (T) (see Definition 5 in [2]). Similarly, we can also define the unstability of the trivial solution of $(\mathrm{N})$ by means of the unstability of the trivial solution of $(\mathrm{T})$. 


\section{AUXILIARY ASSERTIONS}

We determine a matrix norm as a sum of absolute values of the elements of the considered matrix.

AUXILIARY THEOREM. Let us take into account a differential system such that

(2) $\quad \mathbf{C}=\mathbf{x}+\mathbf{D}(t) \mathbf{x}+\mathbf{h}(t, \mathbf{x}), \mathbf{h}(t, \mathbf{o})=\mathbf{o}$, where $\mathbf{C}$ is a constant matrix, (3) $\mathbf{D}(t)$ converges to zero matrix as $t$ tends to infinity and $\mathbf{h}$ is continuous on $(b, \infty) \times A$, where $\quad b \in R, \mathbf{o} \in A \subset R^{4} \quad$ such $\quad$ that

$\|\mathbf{h}(t, \mathbf{x})\| /\|\mathbf{x}\| \rightarrow 0$ uniformly for all $t \geq b$ as $\|\mathbf{x}\| \rightarrow 0$. If there exists an eigenvalue of C having a positive real part, then the null solution of (2) is unstable in Liapunov sense.

PROOF. See [1], Head 13.

Let $u(t)$ solves (E) on $I_{b}$. Let $y(t)$ be an arbitrary function defined on $I_{b}$. Let $z(t)=y(t)-u(t), t \in I_{b}$. Then an arbitrary $z(t)$ solves $(\mathrm{N})$ on $I_{b}$ iff $y(t)$ solves $(\mathrm{E})$ on $I_{b}$. This assertion can be proved in [2, Lemma 1].

The function $u(t)$ solving $(\mathrm{E})$ is stable in Liapunov sense iff 0 solving $(\mathrm{N})$ is stable in Liapunov sense. It is proved in [2, Lemma 1].

\section{RESULTS}

AUXILIARY LEMMA. The function $u(t)$ solving (E) is unstable in Liapunov sense iff 0 solving $(\mathrm{N})$ is unstable in Liapunov sense.

PROOF. The assertion simply follows from the last assertion of the foregoing paragraph.

Now will be proved the unstability criterion of (E).

MAIN THEOREM. Consider (E) such that Main assumption is valid. Let $u(t)$ solve (E) on $I_{b}$ and 


$$
\begin{gathered}
\lim _{t \rightarrow \infty} r_{i}(t)=r_{i} \in(0, \infty), i=1,2,3, \quad \lim _{t \rightarrow \infty} R_{j}(t)=R_{j} \in R, j=0,1,2,3, \\
\frac{|F(t, u(t)+z)-F(t, u(t))|}{|z|} \rightarrow 0 \text { uniformly for all } t \geq b \text { as } z \rightarrow 0 .
\end{gathered}
$$

Let us denote $p(z)=z^{4}+R_{3} z^{3}+\frac{R_{2}}{r_{3}} z^{2}+\frac{R_{1}}{r_{2} r_{3}} z+\frac{R_{0}}{r_{1} r_{2} r_{3}}$. If it holds that

at least one real part of zeros of $p(z)$ is positive,

then $u(t)$ is unstable (in Liapunov sense).

In Main theorem we use the symbols (a), (b') and (c) which are competent to the symbols (a), (b) and (c) in [2].

PROOF OF MAIN THEOREM. From the paragraph “Auxiliary assertions” it is clear that it suffices to check up the unstability of 0 -solution of $(\mathrm{N})$, which correspond to $(\mathrm{E})$; 0 -solution of $(\mathrm{N})$ is unstable iff $(0,0,0,0)$-solution of $(\mathrm{T})$ is unstable. (T) can be expressed as (V), where

$$
\mathbf{z}=\mathbf{C z}+\mathbf{D}(t) \mathbf{z}+\mathbf{h}(t, \mathbf{z}), \quad \mathbf{h}(t, \mathbf{o})=\mathbf{o},
$$

and

$$
\begin{aligned}
& \mathbb{\&}=\left[\begin{array}{c}
\& \\
\& \\
\& \\
\& \\
\&
\end{array}\right], \quad \mathbf{C}=\left[\begin{array}{cccc}
0 & 1 / r_{1} & 0 & 0 \\
0 & 0 & 1 / r_{2} & 0 \\
0 & 0 & 0 & 1 / r_{3} \\
-R_{0} & -R_{1} & -R_{2} & -R_{3}
\end{array}\right], \quad \mathbf{z}=\left[\begin{array}{c}
z_{1} \\
z_{2} \\
z_{3} \\
z_{4}
\end{array}\right], \\
& \mathbf{D}(t)=\left[\begin{array}{cccc}
0 & 1 / r_{1}(t)-1 / r_{1} & 0 & 0 \\
0 & 0 & 1 / r_{2}(t)-1 / r_{2} & 0 \\
0 & 0 & 0 & 1 / r_{3}(t)-1 / r_{3} \\
R_{0}-R_{0}(t) & R_{1}-R_{1}(t) & R_{2}-R_{2}(t) & R_{3}-R_{3}(t)
\end{array}\right], \\
& \mathbf{h}(t, \mathbf{z})=\left[\begin{array}{c}
0 \\
0 \\
0 \\
F\left(t, u(t)+z_{1}(t)\right)-F(t, u(t))
\end{array}\right], \quad \mathbf{o}=\left[\begin{array}{l}
0 \\
0 \\
0 \\
0
\end{array}\right] .
\end{aligned}
$$

If $\|\mathbf{z}\| \neq 0, z_{1} \neq 0$, then 


$$
\begin{gathered}
0 \leq \frac{|| \mathbf{h}(t, \mathbf{z}) \mid}{\| \mathbf{z}||}=\frac{\left|F\left(t, u(t)+z_{1}\right)-F(t, u(t))\right|}{\left|z_{1}\right|+\left|z_{2}\right|+\left|z_{3}\right|+\left|z_{4}\right|}= \\
=\frac{\left|F\left(t, u(t)+z_{1}\right)-F(t, u(t))\right|}{\left|z_{1}\right|} \cdot \frac{\left|z_{1}\right|}{\left|z_{1}\right|+\left|z_{2}\right|+\left|z_{3}\right|+\left|z_{4}\right|} \leq \\
\leq \frac{\left|F\left(t, u(t)+z_{1}\right)-F(t, u(t))\right|}{\left|z_{1}\right|} .
\end{gathered}
$$

If $\|\mathbf{z}\| \neq 0, z_{1}=0$, then $\frac{\|\mathbf{h}(t, \mathbf{z})\|}{\|\mathbf{z}\|}=0$.

From this and (c) it follows that (4) hold. It can be easily found out that (2), (3) hold. It is not difficult to show that $p(z)$ is the characteristic polynomial of $\mathbf{C}$. From (b') it implies that there exists an eigenvalue $a+b i$ of $\mathbf{C}$ such that $a>0$. Then from Auxiliary theorem it implies that $(0,0,0,0)$-solution of $(\mathrm{T})$ is unstable.

EXAMPLE. Consider (E) such that

$$
\begin{gathered}
r_{1}(t)=1+t^{-1}, \quad r_{2}(t)=2+t^{-1}, \quad r_{3}(t)=3+t^{-1}, \\
R_{0}(t)=6+t^{-1}, \quad R_{1}(t)=-24-t^{-1}, \quad R_{2}(t)=18+t^{-1}, \quad R_{3}(t)=-4-t^{-1}, \\
F(t, y)=6 t^{-1}+25 t^{-2}+97 t^{-3}+293 t^{-4}+674 t^{-5}+959 t^{-6}+599 t^{-7}+120 t^{-8}+\left(y-t^{-1}\right)^{2} .
\end{gathered}
$$

It can be taken $b=2$. One can easily seen $t^{-1}$ solves (E) on $I_{2}$ as well as $r_{1}=$ $1, r_{2}=2, r_{3}=3, R_{0}=6, R_{1}=-24, R_{2}=18, R_{3}=-4$. It implies that (a) hold.

If $z \neq 0$, then

$$
\frac{|F(t, u(t)+z)-F(t, u(t))|}{|z|}=\frac{z^{2}}{|z|}=|z| .
$$

From this it implies that $|z|$ uniformly converges to zero for $t \in[2, \infty)$ for $z$ approaching to zero, i.e. (c) hold, too. The characteristic polynomial of $\mathbf{C}$ is $(z-1)^{4}$. One of its zeros is $1+0 i$, i.e. (b') hold. Then, owing to Main theorem, $t^{-1}$ is an unstable solution of considered equation. 


\title{
REFERENCES
}

[1] CODDINGTON, E.A. AND LEVINSON, N. 1955. Theory of ODEs, McGraw-Hill, NY, USA.

[2] PALUMBÍNY, O. 2016. On stability criterion of 4-th order quasilinear differential equations with quasiderivatives, Research papers of Faculty of Materials Science and Technology of Slovak University of Technology in Bratislava, Vol. 24, No. 39, 63-70.

\author{
Oleg Palumbíny \\ UIAM MTF STU \\ J. Bottu 25, 91724 Trnava, Slovakia \\ E-mail: oleg.palumbiny@stuba.sk
}

